\title{
An improved shuffled frog leaping algorithm and its application in permutation flow shop scheduling problem in machine fault conditions
}

\author{
Yueguang $\mathrm{Li}^{1, \mathrm{a}}$ \\ ${ }^{1}$ Gansu Normal University for Nationalities, Hezuo, gansu, 747000, \\ China \\ ${ }^{a}$ Yueguangli7@163.com
}

\begin{abstract}
In order to enhance the performance of shuffled frog leaping algorithm in solving optimization problem, this paper added the mutation operator to original shuffled frog leaping algorithm, an improved shuffled frog-leaping algorithm of solving permutation flow shop scheduling problem was proposed. Simulation results show that the improved algorithm has better feasibility and validity for solving permutation flow shop scheduling problem.
\end{abstract}

Keywords: Shuffled frog leaping algorithm; permutation flow shop scheduling problem; mutation operator; Machine Breakdown

\section{Introduction}

Shuffled frog leaping algorithm is proposed by Muzaffar Eusuff and Kevin Lansey on 2000 year, it is a swarm intelligence computation optimization algorithm ${ }^{[1]}$. It is used to solve discrete combinatorial optimization problem. As a new bionic intelligent optimization algorithm, it combined with memetic algorithms and 
particle swarm optimization algorithm, it has the advantages of two intelligence optimization algorithms. The features of the algorithm are concept simple, parameters is few, calculation speed is fast and global search ability is strong and easy to implement ${ }^{[2]}$. The main application of shuffled frog leaping algorithm is to solve multi objective optimization problems, such as water resources allocation, pier maintenance, workshop arranging flow engineering problem ${ }^{[3]}$. Domestic and foreign scholars have conducted a lot of research on it. Such as: Zhang joins "cognitive component" into the internal search strategy, improve the success rate of the algorithm for solving ability and jump out of local optimal solution ${ }^{[4]}$; Zhao Pengjun joins the attraction repulsion mechanism into the internal search strategy, effectively avoid the algorithm premature convergence ${ }^{[5]}$; Hatem $\mathrm{E}$ in the internal search strategy through the introduction of the "search accelerated factor", to improve the global searching ability of the algorithm ${ }^{[6]}$.

The permutation flow shop scheduling problem is a simplified model of many actual production scheduling problems. Although the process constraint of the model is relatively simple, but it has proven more than 3 machine of permutation flow shop scheduling problem is NP problem ${ }^{[7]}$.

\section{The mathematical model of shuffled frog leaping algorithm}

Shuffled frog leaping algorithm simulates the frog population when they are searching for food, according to ethnic classification of memes of information transfer. Shuffled frog leaping algorithm mainly includes two parts: local search and global information exchange. The following is a brief introduction to the mathematical model of shuffled frog leaping algorithm. 
A randomly generated $\mathrm{F}$ frog consists of initial population, each frog expresses a feasible solution of problem, $U=\left(U^{1}, U^{2}, \ldots, U^{d}\right)$, calculate the frog individual fitness $f(i)$, where $d$ denotes the dimension of the solution space. After the random generation of initial population, the individuals frog according to the fitness $f(i)$ in descending order is stored in the $X=\{U(i), f(i), i=1, \ldots, F\}$, then according to the specific principle to divide the whole frog population into m groups $Y^{1}, Y^{2}, \ldots, Y^{m}$, each group contains the $n$ frogs, satisfy the following relations:

$\left.Y^{K}=\left|U(i)^{k}, f(i)^{k},\right| U(i)^{k}=U(k+m(i-1)), f(i)^{k}=f(k+m(i-1)), i=1, \ldots, n\right] \mid k=1, \ldots, m ; F=m n$

In the frog population, the aim of various group perform local search strategy is to search the local optima in the different search direction, after a certain number of iterations, making the local optimal individuals in the population tends to the global optimal individual.

First of all, the frog population is divided into a plurality of groups, local search is carried out for each ethnic group, in order to avoid the frog individual into a local optimum prematurely, while speeding up the convergence process, in each group, according to specific principles choose a certain number frogs constitute the ethnic sub family group. For the frog population, with global best fitness of the solution is expressed as $U_{g}$; for each sub groups, with the best fitness of the solution is expressed as UB, the worst fitness solutions expressed as UW. The local search is carried out for each sub population, update strategy as following: 


$$
\begin{aligned}
& S= \begin{cases}\min \left\{\left[\operatorname{rand}\left(U_{B}-U_{W}\right)\right], S_{\max }\right\}, & U_{B}-U_{W} \geq 0 \\
\max \left\{\operatorname{rand}\left(U_{B}-U_{W}\right), S_{\max }\right\}, & U_{B}-U_{W}<0\end{cases} \\
& U_{q}=U_{W}+S
\end{aligned}
$$

Among them, $S$ expresses the adjustment vector of frog individual, $S_{\text {mac }}$ represents the maximum step size of the frog allows to change.

Global information exchange helpful to collect local information of all kinds of group search, by meme transmission, obtain the search direction of new global optimal solution. After all populations conduct a certain number of local searches, various groups of frogs are mixed together, according to the fitness of $f(i)$ in descending order, the re division of population, which makes the meme information of the frog individual obtain the full transfer, then continue to conduct local search, so repeatedly until it is convergent, the algorithm stop.

\section{Improvement shuffled frog leaping algorithm for solving permutation flow shop scheduling problem}

Because the standard shuffled frog leaping algorithm, the individual frog position for continuous valued vector, algorithm cannot achieve scheduling updates, so the application of the shuffled frog leaping algorithm for the permutation flow shop scheduling problem needs to be constructed from the individual position to the scheduling appropriate mapping, using reasonable encoding to map the scheduling problem.

According to the characteristics of the permutation flow shop scheduling problem, this paper adopts random keys encoding rules based on the ascending 
order (Ranked Order Value, ROV) ${ }^{[8]}$, the continuous position vector of frog individual $X_{i}=\left(x_{i, 1}, x_{i, 2}, \ldots, x_{i, n}\right)$ turn into the processing sequence $\pi$ of each workpiece on the machine, $\pi=\left(j_{1}, j_{2}, \ldots, j_{n}\right)$, to calculate the corresponding scheduling solution target value of individual frog.

According to the basic convergence properties of shuffled frog leaping algorithm, species formation thought is added in shuffled frog leaping algorithm, proposed an improved shuffled frog leaping algorithm. The specific steps of the algorithm are as follows:

(1) The initialization parameter (the frog population number is $m$, the frog number is $n$ in the population (total number of the frog $F=(m n)$, the frog number is $q$ in sub groups, and the frog update iterations);

(2) Randomly generate F initial feasible solution, and calculate the fitness of the individual frogs;

(3) The frog individual is divided into $m$ groups according to fitness in descending order, construct the sub groups;

(4) Local search. Update the frog individuals for each population group in the sub population groups in accordance with the method of this paper;

(5) Each frog individual mutation, such as the generation of new individuals better than original individual will replace the frog original individual into frog populations, re calculating the degree of adaptation;

(6) Determine whether the algorithm meets convergence conditions, if satisfied, output the optimal scheduling values and the corresponding scheduling solution, return to step (3).

\section{The experimental simulation}

Run environment of algorithm is MATLAB (R2010b) under Win7 operating system. Parameters: the total number of the frog population $F=10$, the number 
of population $m=10$, the number of frog in the sub group $q=2 n / 3$, the iteration number $I T=q$, the biggest adjustment factor number $l_{\max }=n / 2$.

In order to investigate the feasibility and effectiveness of the improved shuffled frog leaping algorithm, this paper chose the RBHFS problems ${ }^{[9]}$, and compared with PSO and IA. All the algorithms are run independently 20 times in each case. The solution of algorithm is shown in table 1 in the case of preempt-resume. In this table, the AVE, MIN and SD respectively represent the average value, the minimum value and variance values. About RBHFS problem, interval mean of all algorithms with 95\%confidence interval of LSD are shown in Figure 1. The smaller the value of ARE, the better the performance of the algorithm.

Table 1 Comparison results on benchmark problems of RBHFS

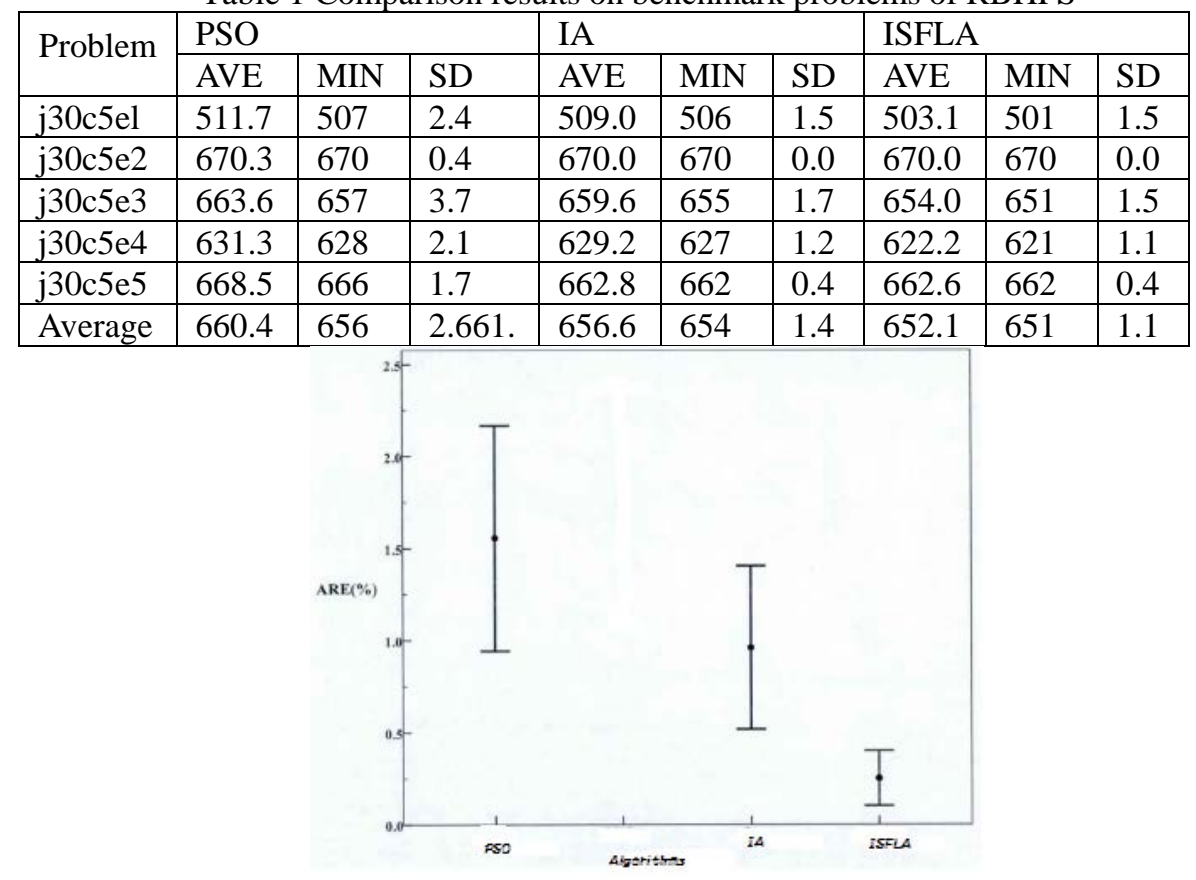

Fig. 1Means plot of ARE with 95\% LSD intervals for different algorithms

\section{Conclusions}


This paper introduced the adjustment sequence of thought on the basis of literature [8], put forward an improved shuffled frog leaping algorithm for solving TSP problem. Simulation results show that improved shuffled frog leaping algorithm in solving the TSP problem has better search performance and robustness.

\section{References}

[1] EUSUFF M M, LANSEY K E. Water distribution network design using the shuffled frog leaping algorithm[A].World Water Congress[C].2001.

[2] LI Y H, ZHOU J Z, YANG J J, et al. Modified shuffled frog leaping algorithm based on threshold selection strategy[J]. Computer Engineering and Applications, 2007, 43(35):19-21.

[3] EUSUFF M M, LANSEY K E. Optimization of water distribution network design using the shuffled frog leaping algorithm[J]. J Water Resource Plan Manage, 2003, 129(3):10-25.

[4] Zhang Xuncai, Hu Xuemei. An improved shuffled frog leaping algorithm with cognitive behavior [C]. 2008.

[5] Zhao Pengjun, Liu Sanyang. The shuffled frog leaping algorithm in solving complex function optimization problems[J]. Application and Research of Computer. 2009, 26(7): 2435 - 2437.

[6]HATEM E, EMAD E, TAREK H, et al. Comparison of two evolutionary algorithms for optimization of bridge deck repairs[J]. Computer-Aided Civil and Infrastructure Engineering, 2006, 21:561-572. 
[7] LUO X H, YANG Y, LI X. Solving TSP with shuffled frog-leaping algorithm [A]. The Eighth International Conference on Intelligent Systems Design and Applications, 2008. 